\title{
Research applications
}

actuator, the scanning speed of the platform is adjustable up to approximately $0.25 \mathrm{~Hz}$. Since the rotating and fixed beamlines are one continuous high vacuum system, a high vacuum rotary union is needed. A non-mechanical, low vapour pressure liquid (ferrofluid) vacuum seal is used. A collimator on the scan arm limits the vertical extent of the neutron beam and there is a fixed collimator in the horizontal plane.

The PFNA concept has undergone numerous tests to demonstrate its efficacy for detecting drugs and explosives. A full-sized system has been built by SAIC in Santa Clara and is undergoing integration testing with a variety of cargoes.

Increase in world trade favours the use of a tool like PFNA cargo inspection for monitoring incoming shipments. PFNA's ability to image elements can be expanded through advanced databases to verify cargo manifests and to monitor shipments. In a modified form, PFNA could also be used to examine the contents of sealed barrels of hazardous waste to avoid manual sampling.

From T.Gozzani, Science Applications International Corporation, Santa Clara, USA

\footnotetext{
Synchrotron radiation is one of the most valuable and widely useful of all accelerator applications. The European Synchrotron Radiation Facility - ESRF - in Grenoble, France is a $6 \mathrm{GeV}$, third generation synchrotron radiation source which will provide up to 30 beamlines for fundamental and applied research.

(Photo ESRF)
}

Most types of accelerator were originally designed by accelerator engineers for particle or nuclear physics research. Today, there are some large and very sophisticated accelerators dedicated to a wide range of other research and development work. Particle accelerators provide a very valuable tool for studying the properties of matter.

\section{Synchrotron radiation research}

n the many varied application fields of accelerators, synchrotron radiation ranks as one of the most valuable and widely useful tools. Synchrotron radiation is produced in multi-GeV electron synchrotrons and storage rings, and emerges tangentially in a narrow vertical fan.

Synchrotron radiation has been

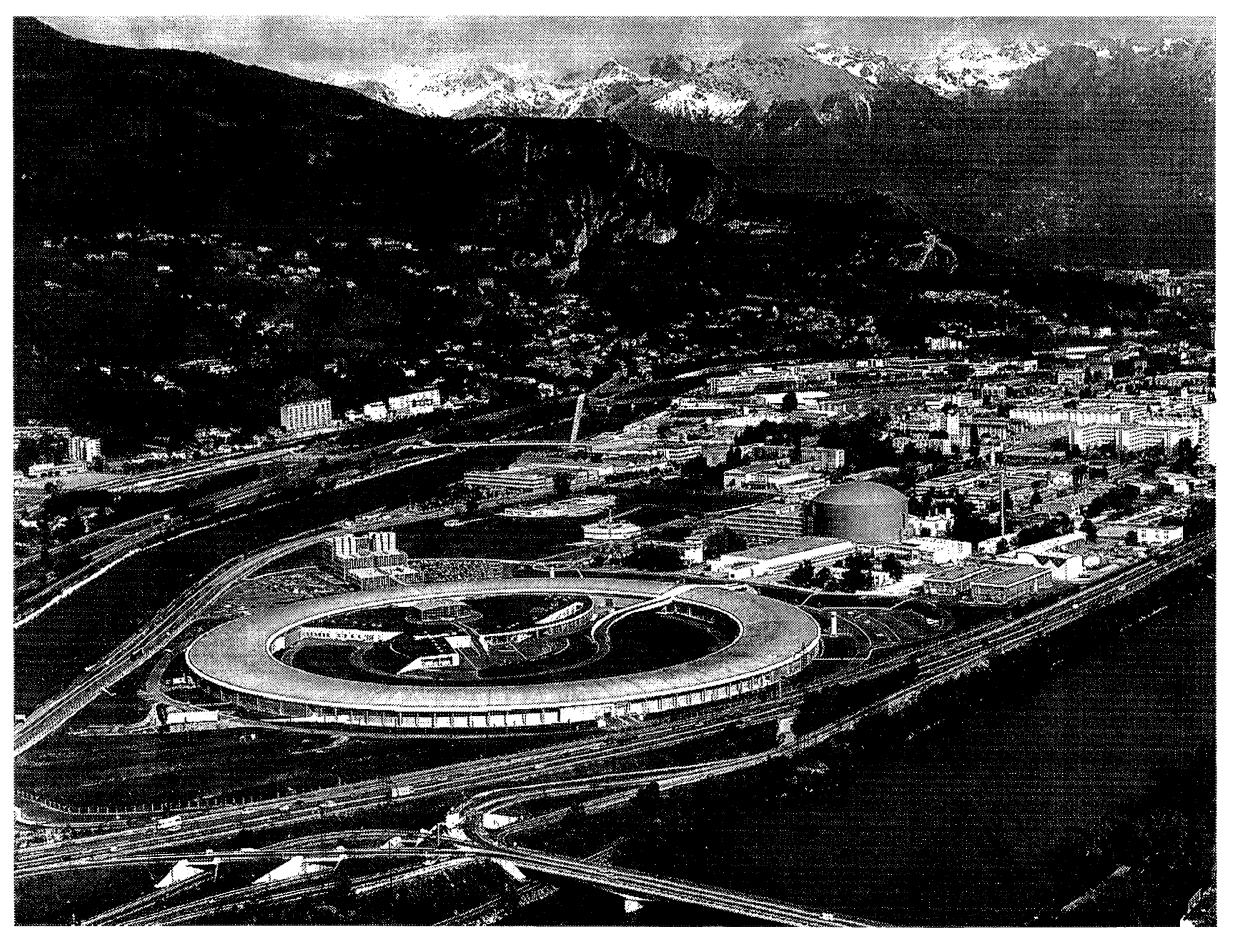

used extensively for basic studies and, more recently, for applied research in the chemical, materials, biotechnology and pharmaceutical industries.

Initially, the radiation was a byproduct of high energy physics laboratories but the high demand soon resulted in the construction of dedicated electron storage rings. The accelerator technology is now well developed and a large number of sources have been constructed, with energies ranging from about 1.5 to $8 \mathrm{GeV}$ including the $6 \mathrm{GeV}$ European Synchrotron Radiation Facility (ESRF) source at Grenoble, France.

A modern third-generation synchrotron radiation source has an electron storage ring with a complex magnet lattice to produce ultra-low emittance beams, long straights for 'insertion devices', and 'undulator' or 'wiggler' magnets to generate radiation with particular properties. Large beam currents are necessary to give high radiation fluxes and long beam 


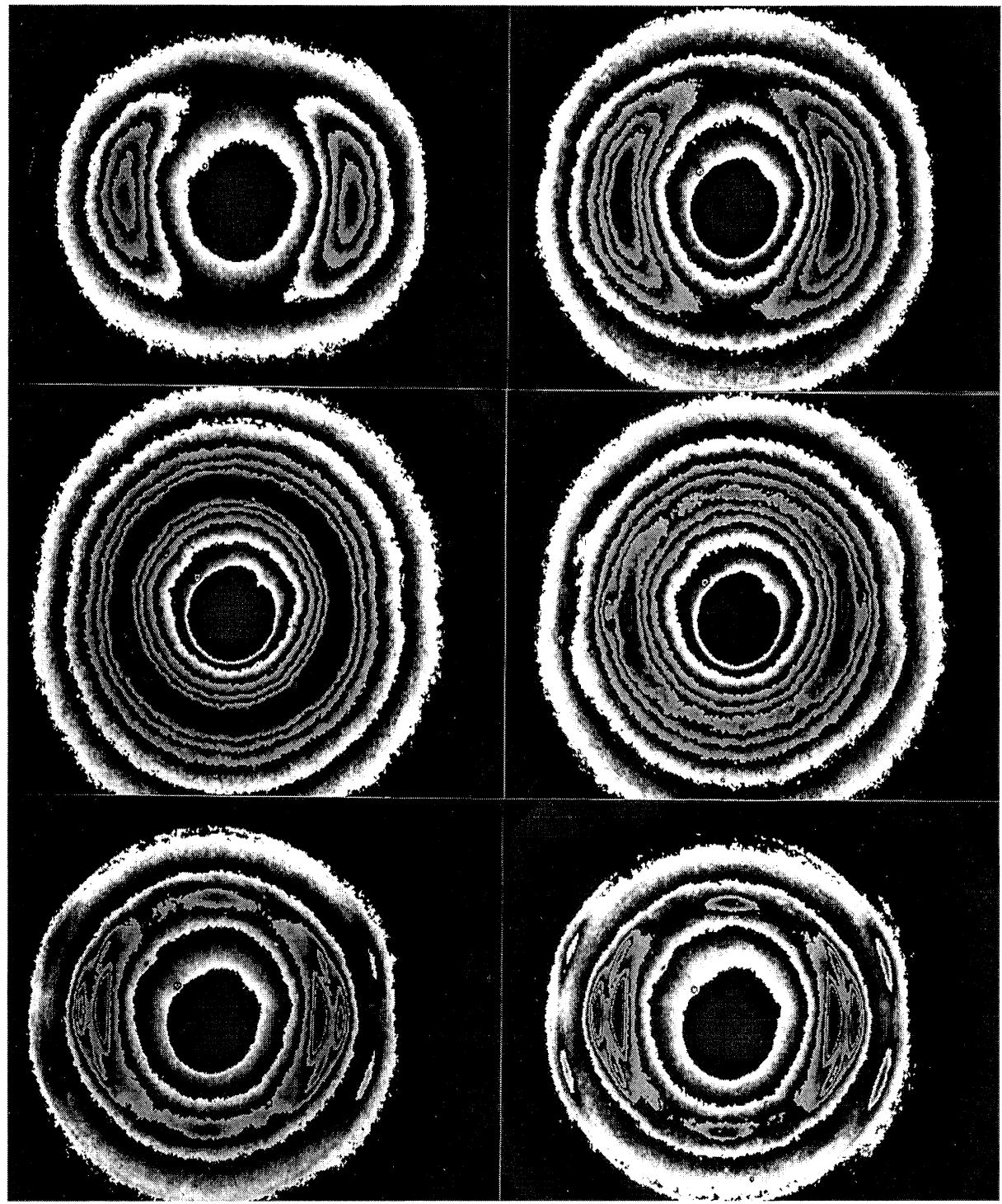

lifetimes require ultra high vacuum systems.

Industrial synchrotron radiation research programmes use either $X$ ray diffraction or spectroscopy to determine the structures of a wide range of materials. Biological and pharmaceutical applications study the functions of various proteins. With this knowledge, it is possible to design molecules to change protein behaviour for pharmaceuticals, or to configure more active proteins, such as enzymes, for industrial processes. Recent advances in molecular biology have resulted in a large increase in protein crystallography studies, with researchers using crystals which, although small and weakly diffracting, benefit from the high intensity. Examples with commercial significance include the study of HIV proteins and inhibitors, the SV40 virus (which can induce tumours), proteins involved in the metabolism of sleeping sickness parasites, and the investigation of xylose isomerase, used industrially to convert sugar to syrup.

Diffraction techniques are widely used in industry for the identification of phases and the analysis of stresses in materials such as thin films. Synchrotron radiation techniques increase the range and complexity of materials that can be studied, such as polymers and thermoplastics, and improve performance. For example Boeing were able to save $30 \%$ in weight in their 757 aircraft, and a larger reduction in cost, by replacing aluminium with glass-filled poly-ether-ether ketone (PEEK) resins.

Using synchrotron radiation, powder diffraction can be used to determine structure and assist in understanding material behaviour, following very rapid transformations. One example is the energy-dispersive rapid diffraction study of the synthesis of zirconia; composite ceramics based on this mineral are used for high temperature mechanical applications, and partially stabilized tetragonal zirconia powder dispersed in a compatible matrix such as alumina or magnesia can impart toughness by arresting micro-crack development. Dynamic "in-situ" studies with synchrotron radiation have shown that crystallization and transformation temperatures depend on the material's thermal history and on the chemical state of the initial zirconium hydroxide. Crystallization and transformation temperatures vary widely, so this information helps chemical engineers obtain the required degree of metastability in zirconia powders for each application.

While X-ray crystallography can be used for well-ordered crystalline substances, other materials often do not have the long range structural order for this technique to be successful. In such cases, X-ray spectroscopy can be effective and $X$ ray absorption in matter and absorption spectra reveal sharp step in- 
creases at certain energies corresponding to the ejection of a bound electron from an atom. In condensed matter there are usually small oscillations superimposed on the top of these edges. These Extended X-ray Absorption Fine Structures (EXAFS), arising from interactions of the outgoing electron with the surrounding atoms, can be analysed to give information on the immediate surroundings. Industrial users of EXAFS facilities at the Daresbury Synchrotron Radiation Source are usually from the chemicals industry, and the work focuses on the behaviour of catalysts.

In the glass industry, the atomic structure of intrinsically disordered systems cannot be prescribed by diffuse diffraction techniques and again EXAFS is a powerful tool, leading to new insights into structure and properties. Combining EXAFS with reflectivity measurements has been important at looking at the surface layers for manufacturers such as Pilkingtons and Schott.

The number of synchrotron sources around the world is increasing rapidly, with two large facilities shortly to become operational in the USA and Japan; elsewhere, smaller sources are planned. The requirement for synchrotron is certain to grow for both academic research and materials development in industry.

From N. Marks, Daresbury and Rutherford Appleton Laboratories

Synchrotron radiation $X$-ray diffraction patterns such as these lead to the improvement of industrial material such as polymers. (Photo ICl plc, UK)

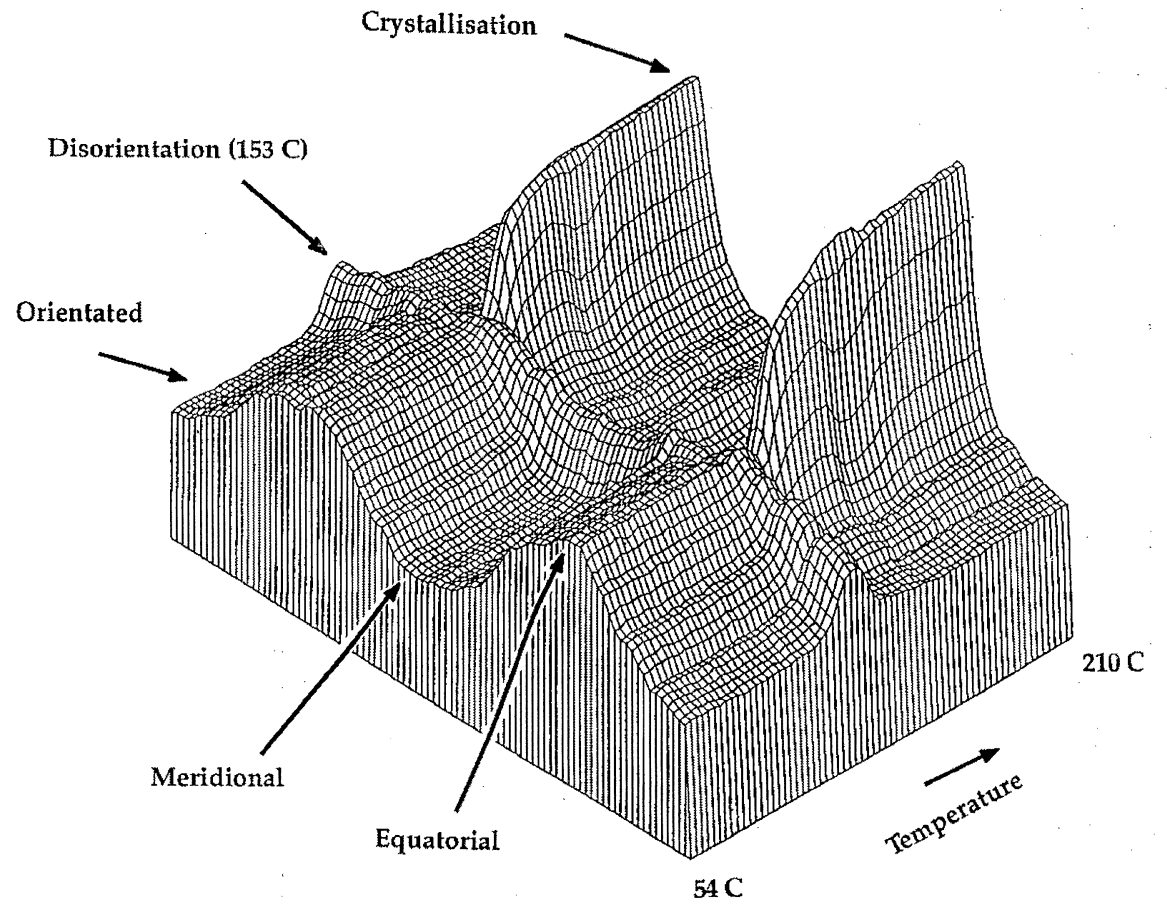

\section{Positron sources}

ormally positron sources are
precursors for accelerator systems in high-energy particle physics; however very low energy positrons are commonly used to study the physics of atomic and condensed matter.

In these experiments low energy, monoenergetic positron beams are produced from high energy positrons by thermalization in special solid moderators. Due to intensity limitations on higher-energy positrons available from radioactive sources, electron linacs have been adapted for this purpose. High energy electron beams bombard thick, high-Z targets, where bremsstrahlung and subsequent pair production create electron-positron pairs. These high energy positrons are injected into a moderator (usually tungsten foils) and emerge at a few eV. The low energy positrons are electrostatically extracted and guided magnetically using low field solenoids. Although the efficiency of the moderation process is around 1 per $10^{6}$, up to $10^{9}$ slow positrons per second have been achieved using linacs with energies up to $100 \mathrm{MeV}$ and currents of 200 microamps.

These high intensity slow positron beams are used for condensed matter studies and atomic physics research, particularly when time correlation is needed between the positron beam and the measured signals or other pulsed devices such as lasers.

In condensed matter research, important results have been obtained from the measurements of the twodimensional angular correlation of annihilation gamma rays of positrons at surfaces from velocity 